\title{
EchoGéo
}

$13 \mid 2010$

Afrique, 50 ans d'indépendance : État et territoires

\section{Sur l'Afrique}

Jean-Louis Chaléard

\section{(2) OpenEdition}

Journals

Édition électronique

URL : https://journals.openedition.org/echogeo/12118

DOI : 10.4000/echogeo. 12118

ISSN : 1963-1197

\section{Éditeur}

Pôle de recherche pour l'organisation et la diffusion de l'information géographique (CNRS UMR 8586)

Référence électronique

Jean-Louis Chaléard, «Sur l'Afrique», EchoGéo [En ligne], 13 | 2010, mis en ligne le 20 septembre 2010 , consulté le 03 août 2021. URL : http://journals.openedition.org/echogeo/12118 ; DOI : https://doi.org/ 10.4000/echogeo. 12118

Ce document a été généré automatiquement le 3 août 2021

EchoGéo est mis à disposition selon les termes de la licence Creative Commons Attribution - Pas d'Utilisation Commerciale - Pas de Modification 4.0 International (CC BY-NC-ND) 


\title{
Sur l'Afrique
}

\author{
Jean-Louis Chaléard
}

1 En cette année 2010, l'Afrique est au cœur de l'actualité. Du cinquantenaire des indépendances pour un grand nombre d'anciennes colonies, notamment françaises, à la coupe de monde de football en Afrique du Sud, l'intérêt pour le continent ne s'est pas démenti tout au long des mois passés. ÉchoGéo, qui a toujours cherché à proposer une réflexion sur des questions du moment, se devait de se pencher sur le continent. Aucune des rubriques habituelles de la revue n'y échappe ! C'est l'occasion de faire le point sur l'évolution souvent heurtée des dernières décennies, de contester des idées reçues, de mettre en exergue les mutations récentes d'une Afrique entrée dans la mondialisation. C'est aussi l'occasion de revenir sur quelques aspects de la production scientifique sur le continent et d'évoquer une grande figure de la géographie africaniste.

2 En effet, la même année où l'Afrique célèbre d'heureux évènements, un géographe passionné de l'Afrique disparait. Nous avons eu l'occasion de souligner dans le précédant numéro combien le décès de Paul Pélissier dans la nuit du 6 au 7 avril 2010 avait affecté les membres de la revue. Nous nous devions de lui rendre hommage et de lui dédier ce numéro, à lui qui a tant aimé et tant défendu les populations africaines. Échogéo a réuni cinq universitaires qui l'ont bien connu : quatre de ses anciens élèves et son successeur comme professeur à l'Université Paris Ouest Nanterre La Défense. La formule retenue, de l'entretien, fait écho à la forme de sa contribution au premier numéro de notre revue en 2007. Si cette formule ne pousse pas à l'analyse exhaustive de l'œuvre, elle a fourni l'occasion aux participants d'évoquer son rôle majeur dans la construction de la géographie africaniste en France et en Afrique, par ses écrits, par les enseignements qu'il a dispensés, par les fonctions qu'il a exercées. Au-delà, les propos recueillis témoignent de l'homme et de la symbiose qui pouvait exister entre son travail intellectuel, son humanisme quotidien, sa manière d'être avec les autres. Géographe exemplaire, modèle pour beaucoup de ses élèves et de ceux qui l'ont côtoyé, il était légitime que cet entretien prenne place dans la rubrique Sur le métier.

3 La rubrique Sur le terrain se concentre sur l'Afrique du Sud. Plusieurs jeunes chercheurs ont contribué au dossier, attestant du dynamisme des recherches en géographie sur ce 
pays, qui fascine à bien des égards. " Puissance émergente et nation adolescente », pour reprendre le titre de Myriam Houssay-Holzschuch, l'Afrique du Sud est complexe et diverse, derrière une vitrine où figurent des personnalités mythiques comme Nelson Mandela. Elle est singulière par son passé marqué par l'apartheid. Comment en sortelle ? Ce pays est un des plus riches (ou le plus riche) du continent, le seul à faire partie $\mathrm{du}$ G20, qui regroupe les grandes puissances économiques du monde et quelques uns des pays émergents les plus dynamiques. Mais la croissance économique ne fait pas disparaître d'un coup toutes les séquelles d'une histoire douloureuse, dans une société qui doit surmonter ses divisions héritées. Les articles s'intéressent surtout aux grandes villes, qui s'insèrent dans le jeu mondial de la métropolisation (Johannesburg, Le Cap, Durban). Ils abordent également quelques thèmes importants et forts du monde actuel, comme les zones de conservation. L'Afrique du Sud devient ainsi un laboratoire pour analyser des questions qui relèvent d'enjeux ou de dynamismes sociaux plus généraux. Quelle est la signification de l'émergence, notion largement économique, dans les champs socio-culturels? Dans quelle mesure l'Afrique du Sud s'approprie-t-elle les modèles venus du Nord? N'en créé-t-elle pas à son tour elle aussi ? Au-delà de son cas particulier, ce pays nous interroge sur l'avenir du continent toute entier et sur les innovations économiques et sociales qui viennent des Suds aujourd'hui.

4 Un certain nombre de thèmes abordés à propos de la «nation arc-en-ciel» se retrouvent dans le dossier de la rubrique Sur le champ, "Afrique, 50 ans d'indépendance. Territoires, frontières, identités et développement » : la place de l'État et des politiques publiques, les dynamiques locales dans la mondialisation, etc. Le dossier appelle l'inventaire. Mais il révèle aussi les profondes transformations que connaît l'Afrique aujourd'hui: Géraud Magrin souligne justement dans son introduction combien sa situation a changé en un quart de siècle tout comme le regard que l'on porte sur elle. Au-delà des incertitudes et des ambiguïtés du moment, le dossier, reprenant cinq décennies de mutations africaines, s'attache à mettre en exergue, les innovations, les mutations, les dynamiques passées et récentes. Il s'interroge sur les bifurcations actuelles de trajectoires qui jusqu'alors étaient largement chaotiques et extraverties.

5 À la suite d'un appel à communication ouvert, la revue a reçu un très grand nombre de propositions pour ce dossier, ce qui témoigne de l'intérêt suscité dans la communauté scientifique par l'Afrique aujourd'hui, alors que les nombreuses manifestations, qui ont lieu tout au long del'année, mobilisent déjà beaucoup de spécialistes français et étrangers. Nous avons été ainsi amenés à composer un dossier beaucoup plus fourni que d'habitude et dont la seconde partie sera publiée dans la prochaine livraison d'ÉchoGéo. Les articles de ce numéro sont consacrés à l'État et aux territoires. C'est l'occasion de mettre l'accent sur les nouvelles politiques publiques, la décentralisation, le rôle du local, etc. C'est aussi l'occasion, de s'interroger sur les différences en ce domaine entre le nord et l'ouest de l'Afrique, et sur les relations parfois paradoxales entre pouvoir central et dynamiques locales.

6 Les rubriques Sur l'Écrit et Sur l'Image parachèvent ce numéro consacré à l'Afrique. Il existe un grand nombre de revues africaines, parfois éphémères, souvent mal connues en France et qui pourtant révèlent la vitalité de la recherche géographique en Afrique et publient des articles dignes d'intérêt. Handicapées par le manque de ressources matérielles et humaines, elles n'ont souvent pas la continuité de grandes revues françaises et leur accès peut être difficile. La recension faite par Bernadette Joseph 
rappelle leur existence et espère susciter l'intérêt pour des textes dans lesquels le lecteur trouvera bien du grain à moudre.

7 Pour faire pendant au dossier sur l'Afrique du Sud, Échogéo propose une analyse de l'ouvrage de P. Gervais-Lambony sur ce pays, paru récemment chez Le Cavalier Bleu, dans la collection Idées reçues. Deux géographes donnent leur point de vue: l'un, français, non spécialiste de l'Afrique, l'autre britannique. La formule nous a paru permettre un éclairage complémentaire, à travers les avis de deux auteurs aux regards différents, l'un " extérieur » au pays mais spécialiste de la discipline en France, l'autre plus « du dedans », mais dont la formation et la façon d'écrire, anglo-saxonnes, ne sont pas celles de la géographie française.

8 Dans la rubrique Sur l'image, la revue innove en consacrant un gros Portfolio à des photographies commentées sur l'Afrique du Sud, qui complète le dossier de Myriam Houssay-Holzschuch sur ce pays. Géographie visuelle du pays qui met en exergue son dynamisme, sa diversité, voire ses contradictions décelables à travers les images et les commentaires qu'en font des géographes spécialistes. C'est l'occasion aussi de rendre un hommage à Benoît Antheaume et Élisabeth Deliry-Antheaume qui ont largement suscité, initié et aidé la recherche française en Afrique du Sud, par leur rôle notamment à l'IFAS (Institut français d'Afrique du Sud).

9 Nous espérons ainsi, dans ce numéro, offrir au lecteur des éléments de réflexion sur l'Afrique actuelle et son devenir, mais aussi sur la recherche africaniste en géographie. Les deux sont liés. Les mutations que l'on peut observer découlent tout autant des modifications du regard que l'on porte que des changements matériels des situations. Une célébration sans complaisance exige un regard de chercheur chaleureux, mais distancié par rapport à la réalité et par rapport à ses propres pratiques. C'est la condition pour présenter, à l'encontre des discours trop souvent simplificateurs, une Afrique plus que jamais plurielle, face aux crises et aux espoirs du moment. 